\title{
Introduction for Volume 8, Issue 4
}

This issue contains 6 papers. There are 2 contributions written in English and 4 contributions in Chinese with English abstracts. The papers can be divided into four topics: health risk, earthquake risk, credit risk and ecological risk.

There are two papers in health risk. The paper "Research on the Occupational Noise Exposure Health Risk Managemnt Standard"by Minyan Li,et al., proposes the procedures and methods of risk assessment and management of occupational noise hazard in the workplaces as technical guidelines in the new standard based on the current status and technical requirements of occupational noise hazard in China. The second paper "The Human Health Risk Assessment Based on Process Simulation and Uncertainty Analysis" by Yue Pan, et al., proposes a human health risk assessment method based on the simulation of contaminant transport process and parameter uncertainty analysis. The method combines health risk assessment with groundwater contaminant transport processes and applies the TOUGH2 program to simulate the temporal and spatial distribution of contaminant in groundwater. The key parameter of the transport model is inversed by Markov chain Monte Carlo simulation method.

There is two papers in earthquake risk. The first paper "Earthquake insurance, catastrophe insurance and earthquake risk \& loss: a comparative analysis based on CiteSpace” by Cong Lang, et al., compared the keyword cluster and institution network of earthquake insurance, other catastrophe insurance and earthquake risk \& loss. The keyword cluster showed that earthquake insurance and other catastrophe study relied on non-engineering approach, such as institutions, policy and risk management. The second paper "A Study of Seismic Macroeconomic Losses Based on Monte Carlo Method- Take Tangshan City as an Example” by Qing Wu, et al., uses the Monte Carlo method to simulate seismic sequences. The model gives the exceeding probability curve of macroeconomic losses.

There is one paper in credit risk. The paper "Collective Risk Generalization to Credit risk" by Reza Habibi, uses the collective risk models of actuarial science, to extend the Creditrisk+ to the case of random number obligors. First, mathematical methods to compute the distribution of total loss are studied. Then, the mathematical results are applied and verified numerically. The insufficiency data in risk management is a big problem. Thus, the case of data scarce is studied using a Bayesian approach. Finally, a concluding remarks section is also given.

There is one paper in ecological risk. The paper "Characteristic Analysis of Ecological Water Use in Xilin River Basin Based on Remote Sensing” by Hongbo Yu, et al., takes the Xilin River basin as the study area and use remote sensing approach to retrieve ecological water use by using MODIS images from from Apr. to Sept. of 2000、2007、2010-2014, and using auxiliary environmental data from the same time periods. The results showed the spatial distribution of ecological water use was consistent with the land surface condition.

We sincerely thank the referees for their strong support and kind help. Thanks to all the authors for their submissions. Particularly, thanks to Prof. Mu Zhang, Publication Chair of the Society for Risk Analysis - China, and thanks to Prof. Junxiang Zhang, Manager of Journal of Risk Analysis and Crisis Response, they devoted their time to overseeing the reviews.

Editors-in-Chief

\author{
Prof. Chongfu Huang \\ Beijing Normal University \\ No.19 Xinjiekouwai Street \\ Beijing 100875, China \\ Email: hchongfu@126.com
}

\author{
Prof. Gordon Huang \\ Faculty of Engineering and Applied Science, \\ University of Regina \\ Regina, Sask S4S 0A2, Canada \\ Email: gordon.huang@uregina.ca
}

